hep-th/0603049

CAS-BHU/Preprint

\title{
An alternative to the horizontality condition in superfield approach to BRST symmetries
}

\author{
R. P. Malik ${ }^{1}$ \\ Centre of Advanced Studies, Physics Department, \\ Banaras Hindu University, Varanasi - 221 005, India
}

\begin{abstract}
We provide an alternative to the gauge covariant horizontality condition which is responsible for the derivation of the nilpotent (anti-)BRST symmetry transformations for the gauge and (anti-)ghost fields of a $(3+1)$ dimensional (4D) interacting 1-form non-Abelian gauge theory in the framework of the usual superfield approach to Becchi-Rouet-Stora-Tyutin (BRST) formalism. The above covariant horizontality condition is replaced by a gauge invariant restriction on the (4,2)-dimensional supermanifold, parameterized by a set of four spacetime coordinates $x^{\mu}(\mu=0,1,2,3)$ and a pair of Grassmannian variables $\theta$ and $\bar{\theta}$. The latter condition enables us to derive the nilpotent (anti-)BRST symmetry transformations for all the fields of an interacting 1-form $4 \mathrm{D}$ non-Abelian gauge theory where there is an explicit coupling between the gauge field and the Dirac fields. The key differences and striking similarities between the above two conditions are pointed out clearly.
\end{abstract}

PACS numbers: 11.15.-q; 12.20.-m; 03.70.+k

Keywords: Horizontality condition, gauge invariant restriction

\footnotetext{
${ }^{1}$ On leave of absence from S. N. Bose National Centre for Basic Sciences, Block JD, Sector - III, Salt Lake, Kolkata - 700 098, West Bengal, India. E-mail addresses : malik@bhu.ac.in ; malik@bose.res.in
} 


\section{Introduction}

The celebrated horizontality condition plays a key role in the usual superfield approach [1-6] to BRST formalism when the latter is applied to the $p$-form $(p=1,2,3, \ldots)$ (non-)Abelian gauge theories. To be more specific and precise, in the framework of the usual superfield approach to a given $D$-dimensional $p$-form Abelian gauge theory, a $(p+1)$-form super curvature $\tilde{F}^{(p+1)}=\tilde{d} \tilde{A}^{(p)}$ is constructed with the help of the super exterior derivative $\tilde{d}=d x^{\mu} \partial_{\mu}+d \theta \partial_{\theta}+d \bar{\theta} \partial_{\bar{\theta}}$ (with $\tilde{d}^{2}=0$ ) and the super $p$-form connection $\tilde{A}^{(p)}$ on a $(D, 2)$-dimensional supermanifold that is parameterized by the $D$-number of commuting spacetime variables $x^{\mu}$ (with $\mu=0,1,2 \ldots D-1$ ) and a pair of anticommuting Grassmannian variables $\theta$ and $\bar{\theta}$ (i.e. $\theta^{2}=\bar{\theta}^{2}=0, \theta \bar{\theta}+\bar{\theta} \theta=0$ ). This super curvature is subsequently equated to the ordinary $(p+1)$-form curvature $F^{(p+1)}=d A^{(p)}$ of the given $D$-dimensional Abelian $p$-form gauge theory which is constructed with the help of the ordinary exterior derivative $d=d x^{\mu} \partial_{\mu}$ (with $d^{2}=0$ ) and the ordinary $p$-form connection $A^{(p)}$. The process of reduction of the $(p+1)$-form super curvature to the ordinary $(p+1)$-form curvature (through the equality $\left.\tilde{F}^{(p+1)}=F^{(p+1)}\right)$ is known as the horizontality condition which has been christened as the soul-flatness condition ${ }^{2}$ by Nakanishi and Ojima [7].

The horizontality condition has also been applied to the physical 1-form non-Abelian gauge theory [3,4] where the super 2-form curvature $\tilde{F}^{(2)}=$ $\tilde{d} \tilde{A}^{(1)}+i \tilde{A}^{(1)} \wedge \tilde{A}^{(1)}$, constructed with the help of the super exterior derivative $\tilde{d}$ and the super 1 -form connection $\tilde{A}^{(1)}$ (by exploiting the MaurerCartan equation), is equated to the ordinary non-Abelian curvature 2-form $F^{(2)}=d A^{(1)}+i A^{(1)} \wedge A^{(1)}$ (where the ordinary exterior derivative $d=d x^{\mu} \partial_{\mu}$ and the ordinary 1 -form connection is $\left.A^{(1)}=d x^{\mu} A_{\mu}\right)$. As is evident from our earlier discussion, the super 2-form curvature $\tilde{F}^{(2)}$ is defined on the (4, 2 )-dimensional supermanifold and the ordinary 2-form curvature $F^{(2)}$ is constructed on the ordinary $4 \mathrm{D}$ spacetime manifold. The key point to be noted is that the horizontality condition is a covariant restriction on the gauge superfield of the $(4,2)$-dimensional supermanifold because the ordinary 2 -form curvature transforms covariantly under the non-Abelian gauge transformation. This condition has been also exploited in the context of usual superfield

\footnotetext{
${ }^{2}$ This condition primarily amounts to setting equal to zero all the Grassmannian components of the $(p+1)$-rank (anti)symmetric curvature tensor that constitutes the $(p+1)$-form super curvature $\tilde{F}^{(p+1)}$. The latter is defined on the (D, 2)-dimensional supermanifold.
} 
approach to BRST symmetries for the gravitational gauge theories [4].

One of the most striking features of the horizontality condition is the fact that it leads to the derivation of the nilpotent (anti-)BRST symmetry transformations for the gauge and (anti-)ghost fields of the Lagrangian density of an interacting non-Abelian gauge theory. It does not shed any light, however, on the derivation of the nilpotent (anti-)BRST symmetry transformations associated with the matter (e.g. Dirac) fields of the above interacting nonAbelian theory. Furthermore, it provides the geometrical origin and interpretations for (i) the existence of the (anti-)BRST symmetry transformations and corresponding (anti-)BRST charges, (ii) the nilpotency property associated with the (anti-)BRST charges (and the (anti-)BRST symmetry transformations they generate), and (iii) the anticommutativity property of the (anti-)BRST charges and corresponding symmetry transformations. These beautiful geometrical interpretations, however, remain confined to only the gauge and (anti-)ghost fields of the (non-)Abelian theories.

The above horizontality condition has recently been augmented [8-16] so that one could derive the nilpotent (anti-)BRST symmetry transformations associated with all the fields of a given (non-)Abelian gauge and/or a reparametrization invariant theories. These extended versions have been christened as the augmented superfield approach to BRST formalism [8-16] where, in addition to the horizontality condition, a set of new restrictions is imposed on the appropriately chosen superfields of the supermanifolds. For instance, one invokes the equality of (i) the conserved quantities [8-13], and (ii) the gauge (i.e. BRST) invariant quantities (that owe their origin to the (super) covariant derivatives [14-17]) in the above extended versions of the usual superfield formalism. The former restriction (in the case of gauge theories and reparametrization invariant theories) leads to a logically consistent derivation $[12,13]$ of the nilpotent symmetry transformations for the matter (or its analogous) fields whereas the latter restriction, for the case of $U(1)$ and $S U(N)$ gauge theories, yields mathematically exact nilpotent symmetry transformations for the matter (e.g. Dirac, complex scalar) fields [14-16]. One of the interesting features of these extensions is the fact that the geometrical interpretations for the (anti-)BRST symmetries and (anti-)BRST charges, found due to the application of horizontality condition alone, remain intact (even in this augmented superfield formalism). However, in all the above endeavours [8-16], one has to exploit both the restrictions (i.e. the horizontality and the additional conditions) separately and independently for 
the derivation of all the nilpotent (anti-)BRST symmetry transformations corresponding to all the fields of the theory.

The purpose of our present paper is to derive the on-shell as well as offshell nilpotent (anti-)BRST symmetry transformations for all the fields of a specific set of Lagrangian densities of a 4D 1-form interacting non-Abelian gauge theory by exploiting a single gauge invariant restriction on the matter superfields of the supermanifolds. In the process, we obtain all the results of the horizontality condition and, on top of it, we obtain the (anti-)BRST symmetry transformations for the matter (Dirac) fields without spoiling the geometrical interpretations of the nilpotent (anti-)BRST symmetry transformations (and corresponding generators) emerging due to the horizontality condition alone. First, as a warm up exercise, we derive the on-shell nilpotent symmetry transformations for all the fields of a given Lagrangian density of the 4D non-Abelian gauge theory by exploiting a gauge invariant restriction on the chiral matter superfields of the $(4,1)$-dimensional chiral supermanifold and pinpoint its striking similarities and key differences with the horizontality condition. Later on, we generalize this discussion to the general supermanifold and derive the off-shell nilpotent (anti-)BRST transformations for all the fields of a given non-Abelian theory. We demonstrate that the gauge (i.e. BRST) invariant restriction on the matter superfields of the supermanifold(s) is superior to the covariant horizontality restriction imposed on those very supermanifold(s). To the best of our knowledge, the BRST invariant restriction, invoked in our present paper, has never been exploited in the context of superfield approach to BRST formalism (except in our earlier paper on the interacting Abelian gauge theory [17]). Thus, our present endeavour is an important step forward in the direction of simplifying and refining the usual superfield approach [1-7] as well as the augmented superfield formalism [8-16] applied to the BRST formulation of the 1-form interacting (non-)Abelian gauge theories.

Our present paper is organized as follows. In section 2, we discuss the bare essentials of the (anti-)BRST symmetry transformations for the 4D 1-form interacting non-Abelian gauge theory in the Lagrangian formulation to set up the notations and conventions. Section 3 is devoted to the derivation of the on-shell nilpotent BRST symmetry transformations for all the fields of the non-Abelian theory by exploiting a gauge (i.e. BRST) invariant restriction on the chiral matter superfields of the $(4,1)$-dimensional chiral super submanifold. The off-shell nilpotent (anti-)BRST symmetry transformations for 
all the fields are derived in section 4 where (i) a general set of superfields are considered on the general $(4,2)$-dimensional supermanifold, and (ii) a gauge (i.e. BRST) invariant restriction is imposed on the matter superfields of the above supermanifold. Finally, in section 5, we make some concluding remarks, point out some key differences between the horizontality condition and our gauge invariant restriction and mention a few future directions for further investigations.

\section{Preliminary: nilpotent symmetry transfor- mations in Lagrangian formulation}

Let us begin with the BRST invariant Lagrangian density of the physical four $(3+1)$-dimensional non-Abelian 1-form interacting gauge theory where there is a coupling between the gauge field and the Dirac fields. This Lagrangian density, in the Feynman gauge, is ${ }^{3}[7,18,19]$

$$
\mathcal{L}_{b}=-\frac{1}{4} F_{\mu \nu} \cdot F^{\mu \nu}+\bar{\psi}\left(i \gamma^{\mu} D_{\mu}-m\right) \psi+B \cdot\left(\partial_{\mu} A^{\mu}\right)+\frac{1}{2} B \cdot B-i \partial_{\mu} \bar{C} \cdot D^{\mu} C
$$

where $F_{\mu \nu}=\partial_{\mu} A_{\nu}-\partial_{\nu} A_{\mu}+i A_{\mu} \times A_{\nu}$ is the field strength tensor for the Lie algebra valued non-Abelian gauge potential $A_{\mu} \equiv A_{\mu}^{a} T^{a}$ that constitutes the 1-form $A^{(1)}$ as: $A^{(1)}=d x^{\mu} A_{\mu}^{a} T^{a}$. Here the generators $T$ 's obey the Lie algebra $\left[T^{a}, T^{b}\right]=f^{a b c} T^{c}$ for a given $S U(N)$ group. The structure constant $f^{a b c}$ can be chosen to be totally antisymmetric in the indices $a, b$ and $c$ for a semi simple Lie group $S U(N)$ [18]. The covariant derivatives $D_{\mu} \psi=\left(\partial_{\mu}+\right.$ $\left.i A_{\mu}^{a} T^{a}\right) \psi$ and $D_{\mu} C^{a}=\partial_{\mu} C^{a}+i f^{a b c} A_{\mu}^{b} C^{c} \equiv \partial_{\mu} C^{a}+i\left(A_{\mu} \times C\right)^{a}$ are defined on the matter (quark) field $\psi$ and ghost field $C^{a}$ such that $\left[D_{\mu}, D_{\nu}\right] \psi=i F_{\mu \nu} \psi$ and $\left[D_{\mu}, D_{\nu}\right] C^{a}=i\left(F_{\mu \nu} \times C\right)^{a}$. It will be noted that these definitions for $F_{\mu \nu}$ agree with the Maurer-Cartan equation $F^{(2)}=d A^{(1)}+i A^{(1)} \wedge A^{(1)} \equiv \frac{1}{2 !}\left(d x^{\mu} \wedge\right.$ $\left.d x^{\nu}\right) F_{\mu \nu}$ that defines the 2-form $F^{(2)}$ which, ultimately, leads to the derivation of $F_{\mu \nu}$. In the above equation (1), $B^{a}$ are the Nakanishi-Lautrup auxiliary

\footnotetext{
${ }^{3}$ We adopt here the conventions and notations such that the Minkowskian 4D metric $\eta_{\mu \nu}=\operatorname{diag}(+1,-1,-1,-1)$ is flat on the spacetime manifold. The dot product and cross product between two non-null vectors $R^{a}$ and $S^{a}$ in the group space of $S U(N)$ are: $R \cdot S=R^{a} S^{a}$ and $(R \times S)^{a}=f^{a b c} R^{b} S^{c}$, respectively. Here the Greek indices $\mu, \nu, \rho \ldots=0,1,2,3$ stand for the spacetime directions on the 4D Minkowski manifold and the Latin indices $a, b, c \ldots .=1,2,3 \ldots$ correspond to the $S U(N)$ group indices.
} 
fields and the anticommuting (i.e. $\left(C^{a}\right)^{2}=\left(\bar{C}^{a}\right)^{2}=0, C^{a} \bar{C}^{b}+\bar{C}^{b} C^{a}=0$, etc.) (anti-) ghost fields $\left(\bar{C}^{a}\right) C^{a}$ are required for the proof of unitarity in the 1-form interacting non-Abelian gauge theory ${ }^{4}$. Furthermore, the $\gamma$ 's are the usual $4 \times 4$ Dirac matrices in the physical 4 D Minkowski space.

The above Lagrangian density (1) respects the following off-shell nilpotent $\left(s_{b}^{2}=0\right)$ BRST symmetry transformations $\left(s_{b}\right)[7,18,19]$

$$
\begin{array}{ll}
s_{b} A_{\mu}=D_{\mu} C, \quad s_{b} C=-\frac{i}{2}(C \times C), & s_{b} \bar{C}=i B, \quad s_{b} B=0, \\
s_{b} \psi=-i(C \cdot T) \psi, & s_{b} \bar{\psi}=-i \bar{\psi}(C \cdot T), \quad s_{b} F_{\mu \nu}=i\left(F_{\mu \nu} \times C\right) .
\end{array}
$$

The on-shell $\left(\partial_{\mu} D^{\mu} C=0\right)$ nilpotent $\left(\tilde{s}_{b}^{2}=0\right)$ version of the above nilpotent symmetry transformations $\left(\tilde{s}_{b}\right)$, are

$$
\begin{array}{lll}
\tilde{s}_{b} A_{\mu}=D_{\mu} C, & \tilde{s}_{b} C=-\frac{i}{2}(C \times C), & \tilde{s}_{b} \bar{C}=-i\left(\partial_{\mu} A^{\mu}\right), \\
\tilde{s}_{b} \psi=-i(C \cdot T) \psi, & \tilde{s}_{b} \bar{\psi}=-i \bar{\psi}(C \cdot T), & \tilde{s}_{b} F_{\mu \nu}=i\left(F_{\mu \nu} \times C\right),
\end{array}
$$

under which the following Lagrangian density

$$
\begin{aligned}
& \mathcal{L}_{b}^{(0)}=-\frac{1}{4} F^{\mu \nu} \cdot F_{\mu \nu}+\bar{\psi}\left(i \gamma^{\mu} D_{\mu}-m\right) \psi \\
& -\frac{1}{2}\left(\partial_{\mu} A^{\mu}\right) \cdot\left(\partial_{\rho} A^{\rho}\right)-i \partial_{\mu} \bar{C} \cdot D^{\mu} C
\end{aligned}
$$

changes to a total derivative (i.e. $\left.\tilde{s}_{b} \mathcal{L}_{b}^{(0)}=-\partial_{\mu}\left[\left(\partial_{\rho} A^{\rho}\right) \cdot D^{\mu} C\right]\right)$. It is straightforward to check that (3) and (4) are derived from (2) and (1), respectively, by the substitution $B=-\left(\partial_{\mu} A^{\mu}\right)$. This relation (i.e. $\left.B=-\left(\partial_{\mu} A^{\mu}\right)\right)$ emerges as the equation of motion from the Lagrangian density (1).

The following off-shell nilpotent $\left(s_{a b}^{2}=0\right)$ version of the anti-BRST $\left(s_{a b}\right)$ transformations (with $s_{b} s_{a b}+s_{a b} s_{b}=0$ ):

$$
\begin{array}{rrr}
s_{a b} A_{\mu} & =D_{\mu} \bar{C}, \quad s_{a b} \bar{C}=-\frac{i}{2}(\bar{C} \times \bar{C}), & s_{a b} C=i \bar{B}, \\
s_{a b} B=i(B \times \bar{C}), & s_{a b} F_{\mu \nu}=i\left(F_{\mu \nu} \times \bar{C}\right), & s_{a b} \bar{B}=0, \\
s_{a b} \psi=-i(\bar{C} \cdot T) \psi, & s_{a b} \bar{\psi}=-i \bar{\psi}(\bar{C} \cdot T), &
\end{array}
$$

\footnotetext{
${ }^{4}$ For the proof of unitarity at a given order of perturbative computation, in the context of a given physical process involving the gauge field and the matter (quark) fields, it turns out that for each bosonic non-Abelian gauge field (gluon) loop diagram, a loop diagram formed by the fermionic (anti-) ghost fields alone, is required (see, e.g. [20]).
} 
are the symmetry transformations for the following equivalent Lagrangians

$$
\begin{aligned}
\mathcal{L}_{\bar{B}}^{(1)} & =-\frac{1}{4} F^{\mu \nu} \cdot F_{\mu \nu}+\bar{\psi}\left(i \gamma^{\mu} D_{\mu}-m\right) \psi+B \cdot\left(\partial_{\mu} A^{\mu}\right) \\
& +\frac{1}{2}(B \cdot B+\bar{B} \cdot \bar{B})-i \partial_{\mu} \bar{C} \cdot D^{\mu} C, \\
\mathcal{L}_{\bar{B}}^{(2)} & =-\frac{1}{4} F^{\mu \nu} \cdot F_{\mu \nu}+\bar{\psi}\left(i \gamma^{\mu} D_{\mu}-m\right) \psi-\bar{B} \cdot\left(\partial_{\mu} A^{\mu}\right) \\
& +\frac{1}{2}(B \cdot B+\bar{B} \cdot \bar{B})-i D_{\mu} \bar{C} \cdot \partial^{\mu} C,
\end{aligned}
$$

where another auxiliary field $\bar{B}$ has been introduced with the restriction $B+$ $\bar{B}=-(C \times \bar{C})$ (see, e.g. [21]). It can be checked that the anticommutativity property $\left(s_{b} s_{a b}+s_{a b} s_{b}=0\right)$ between the (anti-)BRST transformations $s_{(a) b}$ is true for any arbitrary field of the above Lagrangian densities. For the proof of this statement, one should also take into account $s_{b} \bar{B}=i(\bar{B} \times C)$ which is not listed in (2). We emphasize that the on-shell version of the anti-BRST symmetry transformations, to the best of our knowledge, does not exist for all the above cited Lagrangian densities (see, e.g. $[7,18,19]$ ).

All types of nilpotent (of order two) symmetry transformations, discussed and listed above, can be succinctly expressed in terms of the conserved and off-shell nilpotent (anti-)BRST charges $Q_{r}$ and the on-shell nilpotent BRST charge $\tilde{Q}_{b}$, as given below

$$
s_{r} \Sigma=-i\left[\Sigma, Q_{r}\right]_{ \pm}, \quad r=b, a b, \quad \tilde{s}_{b} \tilde{\Sigma}=-i\left[\tilde{\Sigma}, \tilde{Q}_{b}\right]_{ \pm} .
$$

Here the $(+)$ - signs, as the subscripts on the square brackets, stand for the brackets to be the (anti)commutator for the generic field $\Sigma=A_{\mu}, C, \bar{C}, \psi, \bar{\psi}$, $B, \bar{B}$ and $\tilde{\Sigma}=A_{\mu}, C, \bar{C}, \psi, \bar{\psi}$ (present in the above appropriate Lagrangian densities for the 1-form non-Abelian interacting theory) being (fermionic) bosonic in nature. For our discussions, the explicit forms of $Q_{r}(\mathrm{r}=\mathrm{b}, \mathrm{ab})$ and $\tilde{Q}_{b}$ are neither essential nor urgently needed but these can be derived by exploiting the Noether theorem (see, e.g., [7,18,19] for details). 


\section{On-shell nilpotent BRST symmetry transformations: superfield approach}

In this section, first of all, we take the chiral superfields $\mathcal{B}_{\mu}^{(c)}(x, \bar{\theta}), \mathcal{F}^{(c)}(x, \bar{\theta})$, $\overline{\mathcal{F}}^{(c)}(x, \bar{\theta}), \Psi^{(c)}(x, \bar{\theta}), \bar{\Psi}^{(c)}(x, \bar{\theta})$, defined on the $(4,1)$-dimensional super submanifold of the general $(4,2)$-dimensional supermanifold, as the generalization of the basic local fields $A_{\mu}(x), C(x), \bar{C}(x), \psi(x), \bar{\psi}(x)$ of the Lagrangian density (4) which are defined on the $4 \mathrm{D}$ ordinary spacetime manifold. The super expansion of these chiral superfields, in terms of the above basic local fields of the Lagrangian density (4), are as follows

$$
\begin{aligned}
\left(\mathcal{B}_{\mu}^{(c)} \cdot T\right)(x, \bar{\theta}) & =\left(A_{\mu} \cdot T\right)(x)+\bar{\theta}\left(R_{\mu} \cdot T\right)(x), \\
\left(\mathcal{F}^{(c)} \cdot T\right)(x, \bar{\theta}) & =(C \cdot T)(x)+i \bar{\theta}\left(B_{1} \cdot T\right)(x), \\
\left(\overline{\mathcal{F}}^{(c)} \cdot T\right)(x, \bar{\theta}) & =(\bar{C} \cdot T)(x)+i \bar{\theta}\left(B_{2} \cdot T\right)(x), \\
\Psi^{(c)}(x, \bar{\theta}) & =\psi(x)+i \bar{\theta}\left(b_{1} \cdot T\right)(x), \\
\bar{\Psi}^{(c)}(x, \bar{\theta}) & =\bar{\psi}(x)+i \bar{\theta}\left(b_{2} \cdot T\right)(x) .
\end{aligned}
$$

It is evident that, in the limit $\bar{\theta} \rightarrow 0$, we retrieve the basic local fields of the Lagrangian density (4). In the above expansion, there are Lie algebra valued secondary fields $R_{\mu}, B_{1}, B_{2}, b_{1}, b_{2}$ which will be determined, in terms of the basic local fields of the Lagrangian density (4), by the gauge invariant restriction (see, e.g., equation (10) below) on the chiral matter superfields. It will be noted that it is only the matter fields $(\psi(x), \bar{\psi}(x)$ of the Lagrangian density (4) and their chiral superfield generalizations $\Psi^{(c)}(x, \bar{\theta}), \bar{\Psi}^{(c)}(x, \bar{\theta})$ that are not Lie algebra valued. On the r.h.s. of the above expansion, all the fields are well-behaved local fields because they are functions of the $4 \mathrm{D}$ coordinates $x^{\mu}$ alone. Finally, the expansions in (9) are such that the bosonic and fermionic degrees of freedom of the local fields do match. This is an essential requirement for the sanctity of a supersymmetric field theory.

To derive the on-shell nilpotent BRST symmetry transformations (3) for all the local fields, present in the Lagrangian density (4), we begin with the following gauge (i.e. BRST) invariant restriction on the matter chiral superfields of the $(4,1)$-dimensional chiral super sub-manifold:

$$
\bar{\Psi}^{(c)}(x, \bar{\theta}) \tilde{\mathcal{D}}_{\mid(c)} \tilde{\mathcal{D}}_{\mid(c)} \Psi^{(c)}(x, \bar{\theta})=\bar{\psi}(x) D D \psi(x),
$$

where (i) the chiral super sub-manifold is parameterized by four bosonic spacetime coordinates $x^{\mu}(\mu=0,1,2,3)$ and a single Grassmannian variable 
$\bar{\theta}$, (ii) the ordinary covariant derivative $D=d x^{\mu}\left(\partial_{\mu}+i A_{\mu} \cdot T\right.$ ) (on the r.h.s. of (10)) is defined on the ordinary $4 \mathrm{D}$ spacetime manifold, (iii) the chiral super covariant derivative is: $\tilde{\mathcal{D}}_{\mid(c)}=\tilde{d}_{\mid(c)}+i \tilde{A}_{\mid(c)}^{(1)}$. Here the individual terms, present in the definition of chiral super covariant derivative $\tilde{\mathcal{D}}_{\mid(c)}$, are

$$
\tilde{d}_{\mid(c)}=d x^{\mu} \partial_{\mu}+d \bar{\theta} \partial_{\bar{\theta}}, \quad \tilde{A}_{\mid(c)}^{(1)}=d x^{\mu} \mathcal{B}_{\mu}^{(c)}(x, \bar{\theta})+d \bar{\theta} \mathcal{F}^{(c)}(x, \bar{\theta}),
$$

(iv) the explicit computation of the r.h.s. of equation (10), on the ordinary $4 \mathrm{D}$ spacetime manifold, leads to

$$
\begin{aligned}
& \bar{\psi}(x) D D \psi(x)=i \bar{\psi}(x) F^{(2)} \psi(x), \\
& F^{(2)}=\frac{1}{2 !}\left(d x^{\mu} \wedge d x^{\nu}\right)\left(\partial_{\mu} A_{\nu}-\partial_{\nu} A_{\mu}+i A_{\mu} \times A_{\nu}\right),
\end{aligned}
$$

which is a gauge invariant quantity under the $S U(N)$ non-Abelian transformations: $\psi \rightarrow U \psi, \bar{\psi} \rightarrow \bar{\psi} U^{-1}, F^{(2)} \rightarrow U F^{(2)} U^{-1}$ where $U \in S U(N)$, and (v) the definitions (11) are the chiral limit (i.e. $\bar{\theta} \rightarrow 0$ ) of the general expressions for the super exterior derivative $\tilde{d}=d x^{\mu} \partial_{\mu}+d \theta \partial_{\theta}+d \bar{\theta} \partial_{\bar{\theta}}$ and super 1-form connection $\tilde{A}^{(1)}=d x^{\mu} \mathcal{B}_{\mu}(x, \theta, \bar{\theta})+d \theta \overline{\mathcal{F}}(x, \theta, \bar{\theta})+d \bar{\theta} \mathcal{F}(x, \theta, \bar{\theta})$ defined on the general $(4,2)$-dimensional supermanifold (cf. section 4 below).

It is clear from (12) that the r.h.s. of the gauge invariant restriction (10) yields only the coefficient of the 2 -form differential $\left(d x^{\mu} \wedge d x^{\nu}\right)$. The expansion of the l.h.s. would, however, lead to the coefficients of all the possible 2 -form differentials on the $(4,1)$-dimensional chiral super sub-manifold. The explicit form of the expansion, on the l.h.s. of (10), yields

$$
\begin{aligned}
& \left(d x^{\mu} \wedge d x^{\nu}\right) \bar{\Psi}^{(c)}\left(\partial_{\mu}+i \mathcal{B}_{\mu}^{(c)}\right)\left(\partial_{\nu}+i \mathcal{B}_{\nu}^{(c)}\right) \Psi^{(c)}+\left(d x^{\mu} \wedge d \bar{\theta}\right) \\
& \bar{\Psi}^{(c)}\left[\left(\partial_{\bar{\theta}}+i \mathcal{F}^{(c)}\right)\left(\partial_{\mu}+i \mathcal{B}_{\mu}^{(c)}\right)-\left(\partial_{\mu}+i \mathcal{B}_{\mu}^{(c)}\right)\left(\partial_{\bar{\theta}}+i \mathcal{F}^{(c)}\right)\right] \Psi^{(c)} \\
& -(d \bar{\theta} \wedge d \bar{\theta}) \bar{\Psi}^{(c)}\left(\partial_{\bar{\theta}}+i \mathcal{F}^{(c)}\right)\left(\partial_{\bar{\theta}}+i \mathcal{F}^{(c)}\right) \Psi^{(c)}
\end{aligned}
$$

For algebraic convenience, it is advantageous to first focus on the explicit computation of the coefficient of $(d \bar{\theta} \wedge d \bar{\theta})$. This is

$$
-(d \bar{\theta} \wedge d \bar{\theta}) \bar{\Psi}^{(c)}\left[i \partial_{\bar{\theta}} \mathcal{F}^{(c)}-\mathcal{F}^{(c)} \mathcal{F}^{(c)}\right] \Psi^{(c)} .
$$

It is clear from the restriction (10) that the above coefficient should be set equal to zero. For $\Psi^{(c)}(x, \bar{\theta}) \neq 0, \bar{\Psi}^{(c)}(x, \bar{\theta}) \neq 0$, we have the following

$$
\partial_{\bar{\theta}} \mathcal{F}^{(c)}+\frac{i}{2}\left\{\mathcal{F}^{(c)}, \mathcal{F}^{(c)}\right\}=0 .
$$


Substituting the values from the chiral expansion (9) into the above expression, we obtain the following

$$
i B_{1}+\frac{i}{2}(C \times C)+\bar{\theta}\left(C \times B_{1}\right)=0 \Rightarrow B_{1}=-\frac{1}{2}(C \times C), \quad\left(B_{1} \times C\right)=0 .
$$

It is straightforward to note that, not only the condition $\left(B_{1} \times C\right)=0$ is satisfied, we also obtain the BRST transformation $s_{b}$ for the ghost field because the expansion for $\mathcal{F}^{(c)}$ of $(9)$ becomes: $\mathcal{F}^{(c)}(x, \bar{\theta})=C+\bar{\theta}\left(s_{b} C\right)$.

We concentrate now on the explicit computation of the coefficients of the 2 -form differential $\left(d x^{\mu} \wedge d \bar{\theta}\right)$. The final form of this expression is

$$
i\left(d x^{\mu} \wedge d \bar{\theta}\right) \bar{\Psi}^{(c)}\left(\partial_{\bar{\theta}} \mathcal{B}_{\mu}^{(c)}-\partial_{\mu} \mathcal{F}^{(c)}-i\left[\mathcal{B}_{\mu}^{(c)}, \mathcal{F}^{(c)}\right]\right) \Psi^{(c)} .
$$

The restriction in (10) enforces the above coefficient to be zero. This requirement leads to $\left(\right.$ with $\left.\Psi^{(c)}(x, \bar{\theta}) \neq 0, \bar{\Psi}^{(c)}(x, \bar{\theta}) \neq 0\right)$ :

$$
\left(R_{\mu}-D_{\mu} C\right)-i \bar{\theta}\left[D_{\mu} B_{1}+i\left(R_{\mu} \times C\right)\right]=0,
$$

which implies that $R_{\mu}=D_{\mu} C$. Setting equal to zero the $\bar{\theta}$ part of the above equation, entails upon the restriction $D_{\mu}\left[B_{1}+\frac{1}{2}(C \times C)\right]=0$ which is readily satisfied due to the value of $B_{1}$ quoted in (16).

The most important piece of our present computation is the computation of the coefficient of the 2-form differential $\left(d x^{\mu} \wedge d x^{\nu}\right)$ from the 1.h.s. As is evident from (13), with a little bit of algebra, the first term becomes:

$$
\frac{i}{2}\left(d x^{\mu} \wedge d x^{\nu}\right) \bar{\Psi}^{(c)}\left(\partial_{\mu} \mathcal{B}_{\nu}^{(c)}-\partial_{\nu} \mathcal{B}_{\mu}^{(c)}+i\left[\mathcal{B}_{\mu}^{(c)}, \mathcal{B}_{\nu}^{(c)}\right]\right) \Psi^{(c)}
$$

Substituting the explicit expressions for the expansions in (9), we obtain the following form of the above equation

$$
\frac{i}{2}\left(d x^{\mu} \wedge d x^{\nu}\right)\left(\bar{\psi}(x) F_{\mu \nu} \psi(x)+i \bar{\theta}\left[A_{\mu \nu}+i B_{\mu \nu}\right]\right)
$$

where the explicit forms of $A_{\mu \nu}$ and $B_{\mu \nu}$, are

$$
\begin{gathered}
A_{\mu \nu}=\bar{\psi}(x)\left(\partial_{\mu} R_{\nu}-\partial_{\nu} R_{\mu}+i\left[A_{\mu}, R_{\nu}\right]-i\left[A_{\nu}, R_{\mu}\right]\right) \psi(x), \\
B_{\mu \nu}=\bar{\psi}(x) F_{\mu \nu} b_{1} \cdot T+b_{2} \cdot T F_{\mu \nu} \psi(x) .
\end{gathered}
$$


It is straightforward to note that the first term of (20) matches with the r.h.s. of the restriction in (10). With the substitution of $R_{\mu}=D_{\mu} C$, we obtain $A_{\mu \nu}=i\left(F_{\mu \nu} \times C\right)$. Ultimately, setting the $\bar{\theta}$ part of (20) equal to zero, leads to the following relationship ${ }^{5}$

$$
\bar{\psi}(x)\left(F_{\mu \nu} \times C\right) \psi(x)+\bar{\psi}(x) F_{\mu \nu} b_{1} \cdot T+b_{2} \cdot T F_{\mu \nu} \psi(x)=0 .
$$

The above equation can be seen to be readily satisfied if we choose $b_{1} \cdot T=$ $-(C \cdot T) \psi(x)$ and $b_{2} \cdot T=-\bar{\psi}(x)(C \cdot T)$. With the help of these values, it can be seen that the expansion for the matter superfields in (9) become

$$
\begin{aligned}
& \Psi^{(c)}(x, \bar{\theta})=\psi(x)+\bar{\theta}\left(\tilde{s}_{b} \psi(x)\right), \\
& \bar{\Psi}^{(c)}(x, \bar{\theta})=\bar{\psi}(x)+\bar{\theta}\left(\tilde{s}_{b} \bar{\psi}(x)\right) .
\end{aligned}
$$

The above equation provides the geometrical interpretation for the on-shell nilpotent BRST transformation $\tilde{s}_{b}$ (and the corresponding on-shell nilpotent BRST charge $\left.\tilde{Q}_{b}\right)$ as the translational generator $(\partial / \partial \bar{\theta})$ along the Grassmannian direction $\bar{\theta}$ of the $(4,1)$-dimensional chiral supermanifold (cf. equation (8)). In fact, the process of translation of the chiral matter superfields $\Psi^{(c)}(x, \bar{\theta})$ and $\bar{\Psi}^{(c)}(x, \bar{\theta})$ along the Grassmannian direction $\bar{\theta}$ results in the internal BRST transformation $\tilde{s}_{b}$ on the corresponding local matter fields $\psi(x)$ and $\bar{\psi}(x)$ of the Lagrangian density (4) for the ordinary 4D theory.

The above interpretation of the BRST transformation $\tilde{s}_{b}$ (and the corresponding generator $\tilde{Q}_{b}$ ) is valid for all the other superfields of (9). In this connection, it will be noted that we have already computed $B_{1}=-(1 / 2)(C \times C)$ and $R_{\mu}=D_{\mu} C$ from the restriction (10). However, we have not been able to say anything about the secondary field $B_{2}$, present in the expansion of $\overline{\mathcal{F}}^{(c)}$. At this juncture, the equation of motion $B=-\left(\partial_{\mu} A^{\mu}\right)$, derived from

\footnotetext{
${ }^{5}$ It will be noted that the horizontality condition: $\tilde{F}_{\mid(c)}^{(2)}=F^{(2)}$, where $\tilde{F}_{\mid(c)}^{(2)}=\tilde{d} \tilde{A}_{\mid c}^{(1)}+$ $i \tilde{A}_{\mid c}^{(1)} \wedge \tilde{A}_{\mid c}^{(1)}$ and $F^{(2)}=d A^{(1)}+i A^{(1)} \wedge A^{(1)}$, leads to the computation of the l.h.s. as: $(1 / 2)\left(d x^{\mu} \wedge d x^{\nu}\right)\left[F_{\mu \nu}+i \bar{\theta}\left(F_{\mu \nu} \times C\right)\right]$ whereas the r.h.s. is $(1 / 2)\left(d x^{\mu} \wedge d x^{\nu}\right)\left(F_{\mu \nu}\right)$ alone. Here one does not set, the coefficient of $\bar{\theta}$ part of the above equation, equal to zero because that would lead to an absurd result: $\left(F_{\mu \nu} \times C\right)=0$ (which is not the case for our present 4D 1 -form interacting non-Abelian gauge theory). One circumvents this problem by stating that the kinetic energy term $-(1 / 4) F^{\mu \nu} \cdot F_{\mu \nu}$ of the Lagrangian density remains invariant (see, e.g., [3,4]) if $F_{\mu \nu} \rightarrow F_{\mu \nu}+i \bar{\theta}\left(F_{\mu \nu} \times C\right)$. It should be emphasized that such kind of problem does not arise in our present attempt to derive the nilpotent (anti-)BRST symmetry transformations with the gauge invariant restriction (10).
} 
the Lagrangian density (1), comes to our help as we have the freedom to choose $B_{2} \equiv B=-\left(\partial_{\mu} A^{\mu}\right)$. All he above values, finally, imply the following expansions for the chiral superfields defined in (9), namely;

$$
\begin{aligned}
\mathcal{B}^{(c)}(x, \bar{\theta}) & =A_{\mu}(x)+\bar{\theta}\left(\tilde{s}_{b} A_{\mu}(x)\right), \\
\mathcal{F}^{(c)}(x, \bar{\theta}) & =C(x)+\bar{\theta}\left(\tilde{s}_{b} C(x)\right), \\
\overline{\mathcal{F}}^{(c)}(x, \bar{\theta}) & =\bar{C}(x)+\bar{\theta}\left(\tilde{s}_{b} \bar{C}(x)\right),
\end{aligned}
$$

which retain the geometrical interpretation of $\tilde{s}_{b}$ (as well as $\tilde{Q}_{b}$ ) as the translational generator along the Grassmannian direction $\bar{\theta}$ of the chiral supermanifold. It will be noted that this conclusion was also drawn after (24). In other words, the local internal BRST transformations $\tilde{s}_{b}$ for the local basic fields $\left(A_{\mu}(x), C(x), \bar{C}(x)\right)$ of the Lagrangian density (1) is equivalent to the translation of the corresponding chiral superfields $\left(\mathcal{B}_{\mu}^{(c)}(x, \bar{\theta}), \mathcal{F}^{(c)}(x, \bar{\theta})\right.$, $\overline{\mathcal{F}}^{(c)}(x, \bar{\theta})$ along the Grassmannian direction $\bar{\theta}$ of the $(4,1)$-dimensional chiral super sub-manifold of the general $(4,2)$-dimensional supermanifold.

\section{Off-shell nilpotent (anti-)BRST symmetry transformations: superfield formalism}

In this section, we shall derive the off-shell nilpotent symmetry transformations for all the fields of the (anti-)BRST invariant Lagrangian densities (6) and (7) by invoking the same restriction on the matter superfields as quoted in (10) but defined on the general $(4,2)$-dimensional supermanifold:

$$
\bar{\Psi}(x, \theta, \bar{\theta}) \tilde{\mathcal{D}} \tilde{\mathcal{D}} \Psi(x, \theta, \bar{\theta})=\bar{\psi}(x) D D \psi(x),
$$

where all the superfields and super covariant derivatives are parameterized by four $x^{\mu}$ (with $\left.\mu=0,1,2,3\right)$ ) spacetime coordinates and a pair of Grassmannian variables $\theta$ and $\bar{\theta}$. For instance, in the definition of the super covariant derivative $\tilde{\mathcal{D}}=\tilde{d}+i \tilde{A}^{(1)}$, the individual terms are as follows

$$
\begin{aligned}
\tilde{d} & =d x^{\mu} \partial_{\mu}+d \theta \partial_{\theta}+d \bar{\theta} \partial_{\bar{\theta}}, \\
\tilde{A}^{(1)} & =d x^{\mu} \mathcal{B}_{\mu}(x, \theta, \bar{\theta})+d \theta \overline{\mathcal{F}}(x, \theta, \bar{\theta})+d \bar{\theta} \mathcal{F}(x, \theta, \bar{\theta}) .
\end{aligned}
$$

The super expansions for the multiplet fields $\mathcal{B}_{\mu}, \mathcal{F}, \overline{\mathcal{F}}$, in terms of the basic fields $A_{\mu}, C, \bar{C}$ as well as the secondary fields $R_{\mu}, \bar{R}_{\mu}, S_{\mu}, B_{1}, \bar{B}_{1}, B_{2}, \bar{B}_{2}, s, \bar{s}$, 
on the (4,2)-dimensional supermanifold, are [3,4]

$$
\begin{aligned}
\mathcal{B}_{\mu}(x, \theta, \bar{\theta}) & =A_{\mu}(x)+\theta \bar{R}_{\mu}(x)+\bar{\theta} R_{\mu}(x)+i \theta \bar{\theta} S_{\mu}(x), \\
\mathcal{F}(x, \theta, \bar{\theta}) & =C(x)+i \theta \bar{B}_{1}(x)+i \bar{\theta} B_{1}(x)+i \theta \bar{\theta} s(x), \\
\overline{\mathcal{F}}(x, \theta, \bar{\theta}) & =\bar{C}(x)+i \theta \bar{B}_{2}(x)+i \bar{\theta} B_{2}(x)+i \theta \bar{\theta} \bar{s}(x),
\end{aligned}
$$

where all the fields, in the above, are Lie algebra valued. In other words, for the sake of brevity, we have taken the notations $\mathcal{B}_{\mu}=\mathcal{B}_{\mu} \cdot T, B_{1}=B_{1} \cdot T$, etc. In the limit, $(\theta, \bar{\theta}) \rightarrow 0$, we retrieve all the basic local gauge and (anti-)ghost fields of the Lagrangian densities (1), (4), (6) and (7). On the r.h.s. of the above expansion, we can see that the fermionic and bosonic fields (and their degrees of freedom) do match. The super expansions for the fermionic matter superfields $(\Psi(x, \theta, \bar{\theta}, \bar{\Psi}(x, \theta, \bar{\theta})$ in $(26)$, are as follows

$$
\begin{aligned}
& \Psi(x, \theta, \bar{\theta})=\psi(x)+i \theta\left(\bar{b}_{1} \cdot T\right)(x)+i \bar{\theta}\left(b_{1} \cdot T\right)(x)+i \theta \bar{\theta}(f \cdot T)(x), \\
& \bar{\psi}(x, \theta, \bar{\theta})=\bar{\psi}(x)+i \theta\left(\bar{b}_{2} \cdot T\right)(x)+i \bar{\theta}\left(b_{2} \cdot T\right)(x)+i \theta \bar{\theta}(\bar{f} \cdot T)(x),
\end{aligned}
$$

where, it would be noted that, all the secondary fields are Lie algebra valued but the Dirac fields (and corresponding superfields) are not Lie algebra valued as is the case for these fields in the Lagrangian densities.

It is clear that the r.h.s. of (26) (as discussed earlier), is equal to an $S U(N)$ gauge invariant quantity $i \bar{\psi}(x) F^{(2)} \psi(x)$ where the ordinary 2-form $F^{(2)}=\frac{1}{2 !}\left(d x^{\mu} \wedge d x^{\nu}\right)\left(\partial_{\mu} A_{\nu}-\partial_{\nu} A_{\mu}+i A_{\mu} \times A_{\nu}\right)$. The latter contains only a single wedge product of 2 -form differentials (i.e. $\left.\left(d x^{\mu} \wedge d x^{\nu}\right)\right)$ constituted by the spacetime variables alone. However, the l.h.s. would produce all possible differentials of 2 -form defined on the $(4,2)$-dimensional supermanifold. To check this statement, let us first expand the l.h.s. of the gauge invariant restriction (26), in an explicit manner, as

$$
\begin{aligned}
& +\left(d x^{\mu} \wedge d x^{\nu}\right) \bar{\Psi}\left[\left(\partial_{\mu}+i \mathcal{B}_{\mu}\right)\left(\partial_{\nu}+i \mathcal{B}_{\nu}\right)\right] \Psi \\
& -(d \theta \wedge d \theta) \bar{\Psi}\left[\left(\partial_{\theta}+i \overline{\mathcal{F}}\right)\left(\partial_{\theta}+i \overline{\mathcal{F}}\right] \Psi\right. \\
& -(d \bar{\theta} \wedge d \bar{\theta}) \bar{\Psi}\left[\left(\partial_{\bar{\theta}}+i \mathcal{F}\right)\left(\partial_{\bar{\theta}}+i \mathcal{F}\right)\right] \Psi \\
& -(d \theta \wedge d \bar{\theta}) \bar{\Psi}\left[\left(\partial_{\bar{\theta}}+i \mathcal{F}\right)\left(\partial_{\theta}+i \overline{\mathcal{F}}\right)+\left(\partial_{\theta}+i \overline{\mathcal{F}}\right)\left(\partial_{\bar{\theta}}+i \mathcal{F}\right)\right] \Psi \\
& -\left(d x^{\mu} \wedge d \theta\right) \bar{\Psi}\left[\left(\partial_{\mu}+i \mathcal{B}_{\mu}\right)\left(\partial_{\theta}+i \overline{\mathcal{F}}\right)-\left(\partial_{\theta}+i \overline{\mathcal{F}}\right)\left(\partial_{\mu}+i \mathcal{B}_{\mu}\right)\right] \Psi \\
& -\left(d x^{\mu} \wedge d \bar{\theta}\right) \bar{\Psi}\left[\left(\partial_{\mu}+i \mathcal{B}_{\mu}\right)\left(\partial_{\bar{\theta}}+i \mathcal{F}\right)-\left(\partial_{\bar{\theta}}+i \mathcal{F}\right)\left(\partial_{\mu}+i \mathcal{B}_{\mu}\right)\right] \Psi
\end{aligned}
$$

where the anticommutativity property of the matter superfield $\bar{\Psi}$ with the Grassmannian variables $\theta$ and $\bar{\theta}$ has been taken into account. 
For algebraic convenience, it is useful to first compare the coefficients of the differentials $(d \theta \wedge d \theta),(d \bar{\theta} \wedge d \bar{\theta})$ and $(d \theta \wedge d \bar{\theta})$ from the l.h.s. and r.h.s. of the gauge invariant restriction (26). It is obvious that, on the r.h.s., there are no such differentials. Thus, we have to set the above coefficients from the l.h.s. equal to zero. These requirements lead to the following relationships

$$
\begin{aligned}
& \partial_{\theta} \overline{\mathcal{F}}+i \overline{\mathcal{F}} \overline{\mathcal{F}}=0 \Rightarrow \partial_{\theta} \overline{\mathcal{F}}+\frac{i}{2}\{\overline{\mathcal{F}}, \overline{\mathcal{F}}\}=0, \\
& \partial_{\bar{\theta}} \mathcal{F}+i \mathcal{F} \mathcal{F}=0 \Rightarrow \partial_{\bar{\theta}} \mathcal{F}+\frac{i}{2}\{\mathcal{F}, \mathcal{F}\}=0, \\
& \partial_{\theta} \mathcal{F}+\partial_{\bar{\theta}} \overline{\mathcal{F}}+i\{\mathcal{F}, \overline{\mathcal{F}}\}=0,
\end{aligned}
$$

when $\Psi(x, \theta, \bar{\theta}) \neq 0, \bar{\Psi}(x, \theta, \bar{\theta}) \neq 0$. The above conditions lead to the following expressions for the secondary fields in terms of the basic fields:

$$
\begin{aligned}
& \bar{B}_{2}=-\frac{1}{2}(\bar{C} \times \bar{C}), \quad \bar{s}=-i\left(B_{2} \times \bar{C}\right), \\
& \bar{B}_{2} \times \bar{C}=0, \quad \bar{C} \times \bar{s}=i\left(B_{2} \times \bar{B}_{2}\right), \\
& B_{1}=-\frac{1}{2}(C \times C), \quad s=i\left(\bar{B}_{1} \times C\right), \\
& B_{1} \times C=0, \quad(C \times s)=i\left(B_{1} \times \bar{B}_{1}\right), \\
& \bar{B}_{1}+B_{2}=-(C \times \bar{C}), \\
& C \times \bar{s}+s \times \bar{C}=i\left(B_{1} \times \bar{B}_{2}-\bar{B}_{1} \times B_{2}\right), \\
& s=i\left(C \times B_{2}-B_{1} \times \bar{C}\right), \quad \bar{s}=i\left(C \times \bar{B}_{2}-\bar{B}_{1} \times \bar{C}\right) .
\end{aligned}
$$

This equation (i.e. (32)) shows that explicit values of $B_{1}, s, \bar{s}$ and $\bar{B}_{2}$, in terms of the (anti-)ghost fields and auxiliary fields, can be computed and the rest of the above relations are consistent. To see the latter statement clearly, we have to set equal to zero the coefficients of the differentials $\left(d x^{\mu} \wedge d \theta\right)$ and $\left(d x^{\mu} \wedge d \bar{\theta}\right)$. These conditions, for $\Psi \neq 0$ and $\bar{\Psi} \neq 0$, lead to

$$
\begin{aligned}
\partial_{\mu} \overline{\mathcal{F}}-\partial_{\theta} \mathcal{B}_{\mu}+i\left[\mathcal{B}_{\mu}, \overline{\mathcal{F}}\right] & =0, \\
\partial_{\mu} \mathcal{F}-\partial_{\bar{\theta}} \mathcal{B}_{\mu}+i\left[\mathcal{B}_{\mu}, \mathcal{F}\right] & =0 .
\end{aligned}
$$

The outcome of the above conditions is listed below

$$
\begin{aligned}
& R_{\mu}=D_{\mu} C, \quad \bar{R}_{\mu}=D_{\mu} \bar{C}, \quad D_{\mu} \bar{B}_{2}+\bar{R}_{\mu} \times \bar{C}=0 \\
& S_{\mu}=D_{\mu} B_{2}+R_{\mu} \times \bar{C} \equiv D_{\mu} \bar{B}_{1}+\bar{R}_{\mu} \times C, \quad D_{\mu} B_{1}+R_{\mu} \times C=0 \\
& D_{\mu} s=i\left(\bar{R}_{\mu} \times B_{1}-R_{\mu} \times \bar{B}_{1}+S_{\mu} \times C\right), \\
& D_{\mu} \bar{s}=i\left(\bar{R}_{\mu} \times B_{2}-R_{\mu} \times \bar{B}_{2}+S_{\mu} \times \bar{C}\right) .
\end{aligned}
$$


It can be seen explicitly that all the above relationships are consistent with one-another. It is very interesting to pinpoint the fact that the restriction on the auxiliary fields of the Lagrangian densities (6) and (7), advocated by Curci and Farrari (i.e. $B+\bar{B}=-(C \times \bar{C}))$ [21], automatically emerges in our superfield approach if we identify $\bar{B}_{1}=\bar{B}$ and $B_{2}=B$ (cf. (32)).

Finally, we concentrate on the computation of the coefficient of $\left(d x^{\mu} \wedge d x^{\nu}\right)$ from the l.h.s. of the gauge-invariant restriction (26). This can be explicitly expressed, after some algebraic simplification, as

$$
\frac{i}{2}\left(d x^{\mu} \wedge d x^{\nu}\right) \bar{\Psi}(x, \theta, \bar{\theta})\left(\partial_{\mu} \mathcal{B}_{\nu}-\partial_{\nu} \mathcal{B}_{\mu}+i\left[\mathcal{B}_{\mu}, \mathcal{B}_{\nu}\right]\right) \Psi(x, \theta, \bar{\theta}) .
$$

We have to use, in the above, the super expansion of $\mathcal{B}_{\mu}, \Psi, \bar{\Psi}$ from equations (28) and (29). Finally, we obtain the following expression ${ }^{6}$

$$
\frac{i}{2}\left(d x^{\mu} \wedge d x^{\nu}\right)\left[\bar{\psi} F_{\mu \nu} \psi+i \theta L_{\mu \nu}+i \bar{\theta} M_{\mu \nu}+i \theta \bar{\theta} N_{\mu \nu}\right],
$$

where the expressions for $L_{\mu \nu}, M_{\mu \nu}$ and $N_{\mu \nu}$, in full blaze of glory, are

$$
\begin{aligned}
L_{\mu \nu} & =\bar{b}_{2} F_{\mu \nu} \psi-\bar{\psi} F_{\mu \nu} \bar{b}_{1}-\bar{\psi}\left(F_{\mu \nu} \times \bar{C}\right) \psi, \\
M_{\mu \nu} & =b_{2} F_{\mu \nu} \psi-\bar{\psi} F_{\mu \nu} b_{1}-\bar{\psi}\left(F_{\mu \nu} \times C\right) \psi, \\
N_{\mu \nu} & =\bar{f} F_{\mu \nu} \psi+\bar{\psi} F_{\mu \nu} f-i \bar{\psi}\left(F_{\mu \nu} \times \bar{C}\right) b_{1}+i \bar{\psi}\left(F_{\mu \nu} \times C\right) \bar{b}_{1} \\
& +i \bar{\psi}\left[F_{\mu \nu} \times\left(B_{2}+C \times \bar{C}\right)\right] \psi+i \bar{b}_{2} F_{\mu \nu} b_{1} \\
& +i \bar{b}_{2}\left(F_{\mu \nu} \times C\right) \psi-i b_{2} F_{\mu \nu} \bar{b}_{1}-i b_{2}\left(F_{\mu \nu} \times \bar{C}\right) \psi .
\end{aligned}
$$

It is straightforward to check that the first term of (36) does match with the explicit computation of the r.h.s. (i.e. $i \bar{\psi} F^{(2)} \psi$ ) of the gauge invariant restriction (26). This implies immediately that $L_{\mu \nu}, M_{\mu \nu}$ and $N_{\mu \nu}$ must be set equal to zero. It is not very difficult to check that $L_{\mu \nu}=0$ and $M_{\mu \nu}=0$ demand the following expressions for $b_{1}, b_{2}, \bar{b}_{1}, \bar{b}_{2}$; namely,

$$
\bar{b}_{2}=-\bar{\psi}(\bar{C} \cdot T), \quad \bar{b}_{1}=-(\bar{C} \cdot T) \psi, \quad b_{2}=-\bar{\psi}(C \cdot T) \quad b_{1}=-(C \cdot T) \psi .
$$

\footnotetext{
${ }^{6}$ It should be noted that, in the horizontality condition $\tilde{F}^{(2)}=F^{(2)}$, the analogue of (35) from the l.h.s. yields $\frac{i}{2}\left(d x^{\mu} \wedge d x^{\nu}\right)\left[F_{\mu \nu}+i \theta\left(F_{\mu \nu} \times \bar{C}\right)+i \bar{\theta}\left(F_{\mu \nu} \times C\right)-\theta \bar{\theta}\left(F_{\mu \nu} \times B+\right.\right.$ $\left.\left.F_{\mu \nu} \times C \times \bar{C}\right)\right]$. But, the r.h.s. is only $\frac{1}{2}\left(d x^{\mu} \wedge d x^{\nu}\right) F_{\mu \nu}$. One does not set here the $\theta, \bar{\theta}$ and $\theta \bar{\theta}$ parts equal to zero because these lead to absurd results. Rather, one gets rid of this problem by stating that the kinetic energy term $-\frac{1}{4} F_{\mu \nu} \cdot F^{\mu \nu}$ remains invariant under $F_{\mu \nu} \rightarrow F_{\mu \nu}+i \theta\left(F_{\mu \nu} \times \bar{C}\right)+i \bar{\theta}\left(F_{\mu \nu} \times C\right)-\theta \bar{\theta}\left(F_{\mu \nu} \times B+F_{\mu \nu} \times C \times \bar{C}\right)$ (see, e.g., [3,4]).
} 
A few points, regarding the above solutions, are in order. First, a close look at the equation $L_{\mu \nu}=0$ shows that $\bar{b}_{2}$ and $\bar{b}_{1}$ should be proportional to $\bar{\psi}$ and $\psi$, respectively. Second, to maintain the bosonic nature of $\bar{b}_{2}$ and $\bar{b}_{1}$, it is essential that a single fermion should be brought in, together with $\bar{\psi}$ and $\psi$. Finally, the Lie algebra valuedness of $\bar{b}_{2}$ and $\bar{b}_{1}$ requires that $(\bar{C} \cdot T)$ should be brought in for the precise cancellation so that we obtain $L_{\mu \nu}=0$. Precisely similar kinds of arguments go into the determination of the solutions to the equation $M_{\mu \nu}=0$.

Finally, we would like to devote time on finding out the solutions to the condition $N_{\mu \nu}=0$. First of all, it can be seen that we can exploit the values from (38) to simplify $N_{\mu \nu}$. For instance, it can be noted that

$$
-i \bar{\psi}\left(F_{\mu \nu} \times \bar{C}\right) b_{1}-i b_{2}\left(F_{\mu \nu} \times \bar{C}\right) \psi=i \bar{\psi}\left\{F_{\mu \nu} \times \bar{C}, C\right\} \psi \equiv i \bar{\psi}\left(F_{\mu \nu} \times C \times \bar{C}\right) \psi,
$$

and exactly similar kind of counter terms (present in $N_{\mu \nu}$ )

$$
i \bar{\psi}\left(F_{\mu \nu} \times C\right) \bar{b}_{1}+i \bar{b}_{2}\left(F_{\mu \nu} \times C\right) \psi \equiv-i \bar{\psi}\left(F_{\mu \nu} \times C \times \bar{C}\right) \psi,
$$

add to zero. Out of the remaining terms, it can be seen that

$$
i \bar{b}_{2} F_{\mu \nu} b_{1}-i b_{2} F_{\mu \nu} \bar{b}_{1}=-\frac{i}{2} \bar{\psi}\left(F_{\mu \nu} \times C \times \bar{C}\right) \psi .
$$

Thus, ultimately, we obtain the following surviving terms in $N_{\mu \nu}$

$$
\bar{f} F_{\mu \nu} \psi+\bar{\psi} F_{\mu \nu} f+i \bar{\psi}\left(F_{\mu \nu} \times\left(B_{2}+\frac{1}{2} C \times \bar{C}\right)\right) \psi,
$$

which immediately allows us to choose (with identification $B_{2}=B$ )

$$
f=-i\left(B+\frac{1}{2} C \times \bar{C}\right) \psi, \quad \bar{f}=i \bar{\psi}\left(B+\frac{1}{2} C \times \bar{C}\right),
$$

so that $N_{\mu \nu}=0$. Finally, the super expansions in (28) and (29), after insertion of the values from (32), (34), (38) and (43), become

$$
\begin{aligned}
\mathcal{B}_{\mu}(x, \theta, \bar{\theta}) & =A_{\mu}(x)+\theta\left(s_{a b} A_{\mu}(x)\right)+\bar{\theta}\left(s_{b} A_{\mu}(x)\right)+\theta \bar{\theta}\left(s_{b} s_{a b} A_{\mu}(x)\right), \\
\mathcal{F}(x, \theta, \bar{\theta}) & =C(x)+\theta\left(s_{a b} C(x)\right)+\bar{\theta}\left(s_{b} C(x)\right)+\theta \bar{\theta}\left(s_{b} s_{a b} C(x)\right), \\
\overline{\mathcal{F}}(x, \theta, \bar{\theta}) & =\bar{C}(x)+\theta\left(s_{a b} \bar{C}(x)\right)+\bar{\theta}\left(s_{b} \bar{C}(x)\right)+\theta \bar{\theta}\left(s_{b} s_{a b} \bar{C}(x)\right), \\
\Psi(x, \theta, \bar{\theta}) & =\psi(x)+\theta\left(s_{a b} \psi(x)\right)+\bar{\theta}\left(s_{b} \psi(x)\right)+\theta \bar{\theta}\left(s_{b} s_{a b} \psi(x)\right), \\
\bar{\Psi}(x, \theta, \bar{\theta}) & =\bar{\psi}(x)+\theta\left(s_{a b} \bar{\psi}(x)\right)+\bar{\theta}\left(s_{b} \bar{\psi}(x)\right)+\theta \bar{\theta}\left(s_{b} s_{a b} \bar{\psi}(x)\right) .
\end{aligned}
$$


The above expansions, once again, demonstrate the geometrical interpretation of the (anti-)BRST symmetry transformations (and their corresponding generators $\left.Q_{(a) b}\right)$ as the translational generators along the Grassmannian directions $(\theta) \bar{\theta}$ of the $(4,2)$-dimensional general supermanifold. Mathematically, the nilpotency property $\left(s_{(a) b}^{2}=0, Q_{(a) b}^{2}=0\right)$, the anticommutativity property $\left(s_{b} s_{a b}+s_{a b} s_{b}=0, Q_{b}, Q_{a b}+Q_{a b} Q_{b}=0\right)$, etc., can be expressed in terms of the translational generators as

$$
\begin{aligned}
& s_{b} \Leftrightarrow Q_{b} \Leftrightarrow \operatorname{Lim}_{\theta \rightarrow 0} \frac{\partial}{\partial \bar{\theta}}, \quad s_{a b} \Leftrightarrow Q_{a b} \Leftrightarrow \operatorname{Lim}_{\bar{\theta} \rightarrow 0} \frac{\partial}{\partial \theta}, \\
& s_{b}^{2}=0 \Leftrightarrow Q_{b}^{2}=0 \Leftrightarrow\left(\operatorname{Lim}_{\theta \rightarrow 0} \frac{\partial}{\partial \bar{\theta}}\right)^{2}=0, \\
& s_{a b}^{2}=0 \Leftrightarrow Q_{a b}^{2}=0 \Leftrightarrow\left(\operatorname{Lim}_{\bar{\theta} \rightarrow 0} \frac{\partial}{\partial \theta}\right)^{2}=0, \\
& s_{b} s_{a b}+s_{a b} s_{b}=0 \Leftrightarrow Q_{b} Q_{a b}+Q_{a b} Q_{b}=0 \Leftrightarrow \\
& \left(\operatorname{Lim}_{\bar{\theta} \rightarrow 0} \frac{\partial}{\partial \theta}\right)\left(\operatorname{Lim}_{\theta \rightarrow 0} \frac{\partial}{\partial \bar{\theta}}\right)+\left(\operatorname{Lim}_{\theta \rightarrow 0} \frac{\partial}{\partial \bar{\theta}}\right)\left(\operatorname{Lim}_{\bar{\theta} \rightarrow 0} \frac{\partial}{\partial \theta}\right)=0 .
\end{aligned}
$$

This establishes the geometrical interpretations for all the mathematical properties associated with $s_{(a) b}$ and $Q_{(a) b}$.

\section{Conclusions}

One of the central results of our present investigation is the precise derivation of the full set of on-shell as well as off-shell nilpotent (anti-)BRST symmetry transformations associated with all the fields of a given 1-form 4D interacting non-Abelian gauge theory in the superfield formulation. These symmetries emerge from a single gauge (i.e. BRST) invariant restriction (cf. (10) and (26)) on the matter superfields defined on the appropriate supermanifolds. The above restriction is a bold statement that the physical (i.e. BRST invariant) quantities should remain unaltered even in the presence of supersymmetric (Grassmannian) coordinates that appear in the superfield approach to BRST symmetries. This amounts to the requirement that all the wedge products (and otherwise too) of the Grassmannian variables, present in the definition of the above BRST invariant quantities (cf. (10), (26)), should be set equal to zero because the r.h.s. of the above quantities are without them.

The above cited gauge (i.e. BRST) invariant quantities originate from the key properties associated with the (super) covariant derivatives and their 
intimate connections with the definition of the curvature forms on the supermanifolds. Some of the striking similarities and key differences between the horizontality condition and our gauge invariant condition are as follows. First, both of them primarily owe their origin to the (super) cohomological operators $\tilde{d}$ and $d$. Second, the geometrical origin and interpretations for the (anti-)BRST charges (and the nilpotent symmetry transformations they generate) remain intact for the validity of both the conditions on the superfields. Third, whereas the horizontality condition is an $S U(N)$ covariant restriction (because $F^{(2)} \rightarrow U F^{(2)} U^{-1}$ where $U \in S U(N)$ ), the other condition, as the name suggests, is an $S U(N)$ gauge invariant condition. Fourth, the gauge (i.e. BRST) invariant restrictions in (10) and (26) are basically the generalization of the horizontality condition itself where the matter fields (and the corresponding superfields) have been brought into the picture so that these combinations could become the gauge (i.e. BRST) invariant quantities. Finally, there is a very crucial logical (as well as mathematical) difference between the horizontality restriction and the gauge invariant restrictions in (10) and (26). This has been elaborated clearly and cogently in the footnotes before equations (23) and (36) of our present paper.

It is worthwhile to mention that the gauge invariant restrictions in (10) and (26) are superior to (i) the horizontality condition applied in the context of the usual superfield formulation [1-7], and (ii) the consistent extensions of the horizontality condition in the case of the augmented superfield formalism [8-16]. This is due to the fact that (i) whereas the horizontality condition (modulo some logical mathematical questions) leads to the derivation of the nilpotent symmetry transformations for the gauge and (anti-)ghost fields, our gauge invariant restrictions yield all the symmetry transformations for all the fields, and (ii) whereas in the augmented superfield approach, the horizontality condition and the additional restriction(s) are exploited separately and independently, one obtains all the nilpotent (anti-)BRST symmetry transformations for all the fields in one stroke from the gauge invariant restrictions (exploited in (10) and (26) for the appropriately chosen matter superfields).

The highlights of our present endeavour could be enumerated as follows. First of all, the restrictions in (10) and (26) are physically as well as aesthetically more appealing because they are BRST invariant. Second, these gauge (i.e. BRST) invariant restrictions on the superfields are more economical because they produce all the nilpotent symmetry transformations for all the fields of a given 1-form interacting (non-)Abelian gauge theory in one stroke. 
Finally, these restrictions on superfields have very sound mathematical basis at the conceptual level as well as at the algebraic level. Thus, in our entire computation, the thread of logical coherence runs through everywhere.

It would be interesting to extend our prescription (e.g. equations (10) and $(26))$ to a different set of interacting systems so that, the idea proposed in our present investigation, can be put on a firmer footing. For instance, one can check the validity of the analogues of the restrictions (10) and (26) in the context of the interacting $U(1)$ gauge theory where the charged complex scalar fields couple to the $U(1)$ gauge field. It would be more challenging to test the usefulness and sanctity of our idea in the case of gravitational theories (see, e.g., [4] for earlier work) where superfield formulation has been applied to derive the nilpotent (anti-)BRST symmetries. These are some of the immediate issues that are presently under investigation and our results would be reported in our forthcoming future publications [22].

\section{References}

[1] J. Thierry-Mieg, J. Math. Phys. 21, 2834 (1980); Nuovo Cimento A 56, 396 (1980)

[2] M. Quiros, F. J. De Urries, J. Hoyos, M. L. Mozon, E. Rodrigues J. Math. Phys. 22, 1767 (1981)

[3] L. Bonora, M. Tonin M, Phys. Lett. B 98, 48 (1981); L. Bonora, P. Pasti, M. Tonin, Nuovo Cimento A 63, 353 (1981)

[4] R. Delbourgo, P. D. Jervis, J. Phys. A: Math. Gen. 15, 611 (1981); R. Delbourgo, P. D. Jervis, G. Thompson, Phys. Lett. B 109, 25 (1982)

[5] L. Baulieu, J. Thierry-Mieg, Nucl. Phys. B 197, 477 (1982); Nucl Phys. B 228, 259 (1982); L. Alvarez-Gaumé, L. Baulieu, Nucl. Phys. B 212, 255 (1983)

[6] D. S. Hwang, C. -Y. Lee, J. Math. Phys. 38, 30 (1997)

[7] N. Nakanishi, I. Ojima, Covariant Operator Formalism of Gauge Theories and Quantum Gravity (World Scientific, Singapore, 1990)

[8] R. P. Malik, Phys. Lett. B 584, 210 (2004) [hep-th/0311001] 
[9] R. P. Malik, Int. J. Geom. Methods Mod. Phys. 1, 467 (2004) [hepth/0403230]

[10] R. P. Malik, J. Phys. A: Math. Gen. 37, 5261 (2004) [hep-th/0311193]

[11] R. P. Malik, Int. J. Mod. Phys. A 20, 4899 (2005) Erratum ibid. A 20, 7285 (2005) [hep-th/0402005]

[12] R. P. Malik, Mod. Phys. Lett. A 20, 1667 (2005) [hep-th/0402123]

[13] R. P. Malik, Eur. Phys. J. C 45, 513 (2006) [hep-th/0506109]

[14] R. P. Malik, Eur. Phys. J. C 47, 227 (2006) [hep-th/0507127]

[15] R. P. Malik, Eur. Phys. J. C 48, 825 (2006) [hep-th/0510226]

[16] R. P. Malik, B. P. Mandal, Eur. Phys. J. C 47, 219 (2006) [hepth/0512334]

[17] R. P. Malik, J. Phys. A: Math. Gen. 39, 10575 (2006) [hep-th/0510164]

[18] S. Weinberg, The Quantum Theory of Fields: Modern Applications, Vol. 2 (Cambridge University Press, Cambridge, 1996)

[19] K. Nishijima, Czech. J. Phys. 46, 1 (1996)

[20] I. J. R. Aitchison, A. J. G. Hey, Gauge Theories in Particle Physics: A Practical Introduction (Adam Hilger, Bristol, 1982)

[21] G. Curci, R. Ferrari, Phys. Lett. B 63, 51 (1976); Nuovo Cimento A 32, 151 (1976)

[22] R. P. Malik, in preparation. 